ARTICLE

\title{
Management of patients with chronic kidney disease
}

T Gerntholtz, ${ }^{1}$ FCP (SA); G Paget, ${ }^{2} \mathrm{MB}$ BCh, FCP (SA), Cert Nephrology (SA), MMed; P Hsu, ${ }^{3}$ MB BCh, FCP (SA);

A M Meyers, ${ }^{4}$ MB BCh, FCP (SA), Cert Nephrology (SA), FRCP (Lond)

${ }^{1}$ Life Fourways Hospital, Johannesburg, South Africa

${ }^{2}$ Division of Medicine (Nephrology), Faculty of Health Sciences, University of the Witwatersrand, and Charlotte Maxeke Johannesburg Academic Hospital, Johannesburg, South Africa

${ }^{3}$ Netcare Milpark Hospital, Johannesburg, South Africa

${ }^{4}$ Donald Gordon Medical Centre, Klerksdorp Hospital, and National Kidney Foundation of South Africa, Johannesburg, South Africa

Corresponding author: A M Meyers (nkfsa@mweb.co.za)

Co-operation between primary healthcare workers (clinic staff and general practitioners) and nephrologists is essential and the ability to refer patients timeously should be on a pre-negotiated and organised basis. This article deals with these aspects, including follow-up guidelines and management and treatment strategies, including lifestyle changes where indicated and referral for end-stage renal failure, i.e. for dialysis and transplantation.

S Afr Med J 2015;105(3):237. DOI:10.7196/SAMJ.9417

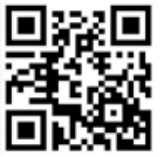

Referral of patients to a nephrologist

It is crucial to establish a trusting and co-operative relationship between the primary care practice/ unit and nephrologist, with easy access between both. Many patients with chronic kidney disease (CKD) can be sufficiently managed in-between infrequent nephrological consultations.

It is important to first screen those at risk for kidney disease, including those with:

- hypertension

- diabetes

- HIV infection

- a family history of kidney disease

- a clinical picture compatible with kidney disease.

In the abovementioned cases, those with the following clinical characteristics would require timeous referral:

- urinary abnormalities on dipstick testing

- haematuria

- proteinuria $(>1+)$; this should be measured using a spot urine albumin or protein to creatinine ratio (first morning void preferred but not essential)

- reduced glomerular filtration rate (GFR), i.e. stage 3 - 5 CKD (GFR $<30-60 \mathrm{~mL} / \mathrm{min}$ ) or with rapidly rising serum creatinine

- resistant hypertension, typically requiring $\geq 3$ antihypertensive agents

- any noticeable or significant changes in their clinical or histological status.

It is essential that all intervening blood and urine results are communicated to both a nephrologist and primary care practice. Timeous referral to a nephrologist will allow:

- management of the complications of CKD, e.g. renal anaemia, bone mineral disease, hypertension

- preparation for the appropriate renal replacement therapy, i.e. transplantation v. dialysis, either peritoneal or haemodialysis

- insertion of catheters and fistulas

- exploration of funder options, e.g. state or private.

\section{Follow-up of CKD patients in South Africa Importance of CKD follow-up}

Worldwide, CKD is believed to affect $10 \%$ of the population; so one could expect around 500000 people to be affected in South Africa (SA).

SA has very high prevalence rates of three important diseases that put people at risk of $\mathrm{CKD}$, i.e. hypertension, type 2 diabetes mellitus and HIV.

Prevention of progression of CKD is best achieved earlier than later.

Delay of progression to end-stage kidney disease (ESKD) is particularly important in a resource-limited setting such as SA, where access to dialysis is limited by suitability for renal transplantation, particularly in the state sector.

\section{Identifying patients who need follow-up and follow-up strategies}

Those most at risk should be screened annually by assessing GFR and urinary protein (this can be easily performed on spot urine protein or albumin/creatinine ratios). Patients with the following are included:

- hypertension

- diabetes

- HIV infection

- vascular disease (coronary and peripheral)

- advanced age, especially bone pain and proteinuria in the elderly (myeloma is quite common)

- a family history of kidney disease

- a recurring urinary tract infection (especially in children)

- nephrotoxic drug use - non-steroidal anti-inflammatory drugs (NSAIDs), lithium and tenofovir.

One should consider referral to a nephrologist for an opinion in the following situations:

- Proteinuria, especially $>1 \mathrm{~g} /$ day or worsening proteinuria and/or persistent haematuria, as these may indicate glomerulonephritis.

- GFR $<60 \mathrm{~mL} / \mathrm{min}$ or GFR worsening rapidly (acutely or $>5 \mathrm{~mL} /$ year).

- All children should be referred.

- Familial kidney diseases, e.g. polycystic kidney disease. 
Most patients can be reviewed annually, but consider more frequent visits/investigations in the following situations:

- When patients are ill or have to undergo surgery.

- Poor blood pressure (BP) control, provided the patient is compliant. Aim to maintain BP $<130 / 80 \mathrm{mmHg}$.

- Poor control of diabetes (glycated haemoglobin $\left(\mathrm{HbA}_{1 \mathrm{C}}\right)>8 \%$ ). Beware of increased potency of hypoglycaemic drugs at lower GFR.

- $\mathrm{GFR}<30 \mathrm{~mL} / \mathrm{min}$

- Significant comorbidities (especially cardiac disease).

\section{Types and frequency of investigations in CKD}

- BP should be checked at each visit.

- Proteinuria and creatinine should be checked at least annually, but more frequently in those with glomerular disorders and progressive CKD.

- Renal imaging should be performed as a screening test on every person with CKD. It may need to be repeated to screen for malignancies and when a sudden decline in renal function occurs to rule out obstruction.

- Haemoglobin $(\mathrm{Hb})$ should be checked at least annually, but needs to be reviewed at least every 3 months when GFR $<30 \mathrm{~mL} / \mathrm{min}$. In anaemic patients, it is important to check iron status before starting erythropoietin therapy - try to maintain $\mathrm{Hb} 11-12 \mathrm{~g} / \mathrm{dL}$.

- In all patients with GFR $<30 \mathrm{~mL} / \mathrm{min}$ it is important to monitor secondary hyperparathyroidism - check corrected serum calcium and phosphate. Check parathyroid hormone at least 6-monthly.

- Monitoring of serum potassium is also important in CKD patients, especially those on angiotensin-converting enzyme (ACE) inhibitors and with a lower GFR $(<30 \mathrm{~mL} / \mathrm{min})$.

- Remember comorbid conditions - patients with diabetes need cardiovascular review, including stress and pulse rate testing, review of foot health and retinopathy/cataracts at least once a year; those with ischaemic heart disease also require cardiac review at least once a year.

\section{Remember that cardiovascular mortality is high in cases of CKD.}

\section{Conservative management of CKD} patients in SA

\section{Importance of management of CKD}

- CKD management is particularly important to prevent or delay progression to ESKD in a resource-limited setting such as SA, where the state will only chronically dialyse patients suitable for transplantation.

- Seeing patients earlier in the course of ESKD, allows better planning for renal replacement therapy (RRT) should the need arise. Patients not eligible for state dialysis programmes will have waiting periods for cover for RRT imposed by medical aids for up to 12 months. Patients may also be able to undergo transplantation before needing dialysis.

\section{Aspects of management of CKD}

- Lifestyle and diet. These will be covered in the next section.

- Hypertension. Aim to achieve consistent BP $<130 / 80 \mathrm{mmHg}$. Multiple drugs may be required. ACE inhibitors and angiotensin II receptor blockers (ARBs) are useful agents, particularly in proteinuric patients, but need to be used with care in the elderly and in those with vasculopathy and advanced CKD. Refer to the hypertension guideline for use of antihypertensive agents for multiple therapeutic purposes. ${ }^{[1]}$
- Proteinuria. There is currently little and controversial evidence for the use of ACE inhibitors and ARBs as agents to prevent the onset of albuminuria and progression of renal disease in patients with microalbuminuria other than in hypertensive nephrosclerosis. However, in diabetes or chronic non-diabetic glomerulonephritis with overt proteinuria evidence supports the use of ACE inhibitors or ARBs to prevent or delay progression of CKD. Currently, evidence for the combined use of these agents to delay progression is controversial (see results of the ONTARGET study ${ }^{[2]}$ ). Overall control of BP to at least $130 / 80 \mathrm{mmHg}$ is perhaps the most important aspect of control of proteinuria, regardless of the agents used. Note that non-dihydropyridine calcium channel blockers such as verapamil also have antiproteinuric properties and act synergistically with ACE inhibitors or ARBs. Of considerable importance is review of the use of ACE inhibitors and ARBs in patients with stages 4 and $5 \mathrm{CKD}$ due to the possible production of potentially dangerous electrolyte charges or sudden and permanent end-stage renal failure (ESRF). This is of particular importance in the elderly and diabetics.

- Diabetes. In both type 1 and 2 diabetes evidence exists that good control $\left(\mathrm{HBA}_{1 \mathrm{c}}<7 \%\right)$ of glucose has a beneficial effect on progression and prevention of CKD, particularly of other microvascular complications.

- Anaemia. This will be covered in more detail in the April 2015 edition of CME. Maintenance of $\mathrm{Hb} 10-12.5 \mathrm{~g} / \mathrm{dL}$ is optimal and improves quality of life. However, published evidence does not support the notion that control of anaemia delays progression or improves cardiovascular mortality. Care should be exercised with therapy so as not to exceed $\mathrm{Hb} 13 \mathrm{~g} / \mathrm{dL}$.

- Planning RRT. This is an often neglected area of CKD management. It is acceptable and ideal below a GFR $20 \mathrm{~mL} / \mathrm{min}$ to consider and arrange access for dialysis, or ideally pre-emptive kidney transplantation.

- Avoidance of nephrotoxic procedures and medications. This is a particularly important area of CKD management. Thought should be given to the real risk/benefits ratio of contrast procedures. Patients should be educated to discuss the advisability of continuing medications such as diuretics, certain antihypertensives and metformin with their doctors when they are ill or have to undergo surgery. CKD patients need to be warned to avoid NSAIDs, certain combination analgesics and some herbal medications that may lead to acute renal failure.

\section{Modification of lifestyle and diet for CKD patients in SA Importance of lifestyle/behaviour}

- Prevention of progression of CKD. There is good evidence that practices such as smoking can accelerate progression to ESKD. Obesity may induce renal disease and/or diabetes.

- Prevention of cardiovascular morbidity/mortality. It is well known that CKD patients are at high risk of cardiovascular disease, which is exacerbated by being centrally obese, smoking or having poor control of diabetes and hypertension.

- Eligibility for RRT. This is a somewhat uniquely SA issue. Severe obesity (body mass index (BMI) $>35 \mathrm{~kg} / \mathrm{m}^{2}$ ), smoking and abuse of substances such as alcohol, may preclude patients from state dialysis and transplantation programmes.

\section{Aspects of lifestyle and diet}

- Smoking cessation is important to avoid the progression of CKD and the development of cardiovascular disease.

- Ideally, keep caloric intake to 30 - $35 \mathrm{kcal} / \mathrm{kg} / \mathrm{day}$, although if overweight, reduce it to $25 \mathrm{kcal} / \mathrm{kg} /$ day. 
- In earlier stages of CKD (3 - 5) protein restriction of $0.8 \mathrm{~g} / \mathrm{kg} /$ day may be beneficial to avoid progression of CKD. Protein restriction is not advisable for dialysis patients, who should eat protein $1-2 \mathrm{~g} / \mathrm{kg} /$ day.

- Fluid intake should exceed urine output by around $500 \mathrm{~mL} /$ day, or patients can be advised to weigh themselves daily to titrate intake/diuretic doses/fluid restriction against their established dry weight. Patients with cardiac failure may need more aggressive fluid restriction. Patients should be cautioned to reduce diuretic doses when fluid losses are high, e.g. sweating in hot environments, gastroenteritis.

- It is unhealthy to consume more than 14 units of alcohol per week for males, or 7 units per week for females.

- Salt restriction helps to control thirst and maintain dry weight (and BP). Ideally, patients should add no salt when cooking food or to any food. Salt intake should be restricted to $1-2 \mathrm{~g} /$ day.

- It is advisable to keep potassium intake at $2-3 \mathrm{~g} /$ day. Foods to be avoided are potatoes (soaking overnight in water will remove potassium), dried fruits, tomatoes, bananas, nuts and sweets. It is best to refer patients to a dietitian to discuss their diets.

- In terms of calcium and phosphate, patients should be advised to limit the intake of high calcium foods (dairy products), and avoid vascular and other ectopic calcification, especially when using calcium-containing phosphate binders. Once the GFR falls below $\sim 30 \mathrm{~mL} / \mathrm{min}$, it is important that patients are advised to limit phosphate in the diet (e.g. bran, brown rice, dried beans, lentils, offal, salmon, chocolate, cola drinks and milk products).

\section{RRTs - dialysis, transplantation and organ donation}

RRTs place a great financial burden on our healthcare system. The demand for dialysis and transplantation greatly outweighs the supply of available facilities. This is essentially why early preventive therapy is vitally important to delay and minimise progression of renal disease.

For those with ESKD, timeous referral for assessment for transplantation is vital. Increased non-related living donation, related living donation and pre-emptive transplantation will improve quality of life and increase available dialysis slots to accommodate new ESKD patients.

In SA there is a significant number of patients suffering from ESKD, especially among the African population (as a result of malignant hypertension). HIV infection has also increased the number of patients requiring RRT, as it is a cause of CKD.

All patients suffering from ESKD should be considered for RRT, which includes:

- peritoneal dialysis

- automated peritoneal dialysis

- haemodialysis

- renal transplantation.

\section{Dialysis}

There are few contraindications to dialysis, the main concern usually being quality of life and survival. Patients with the following chronic illnesses will probably not benefit from dialysis, as mortality will be high:

- severe cardiomyopathy with refractory cardiac failure

- advanced liver cirrhosis

- disseminated, terminal malignancy

- end-stage chronic obstructive pulmonary disease

- severe dementia

- debilitating cerebrovascular accident.
Regrettably, in SA, financial constraints often play an important role in determining the availability of dialysis. We should endeavour to offer all our patients appropriate RRT, as the alternative is unthinkable. Nevertheless, currently probably only about $10 \%$ of those who would benefit from dialysis are receiving this therapy. In the past 10 years, the economy of SA has grown and the availability of dialysis centres should likewise have improved. This is, however, questionable but nonetheless all patients with ESKD should at least be referred to a renal facility for assessment.

\section{Transplantation and renal donation}

The number of patients awaiting renal transplantation has increased over time. The waiting time has increased owing to widening of the gap between allograft supply and demand.

There is an urgent need to increase the donor pool, potentially from:

- deceased donors

- living related donors

- living non-related donors.

All provinces have organ transplant co-ordinators who should be contacted for all potential deceased donors. A complete list of co-ordinators is available from the Organ Donor Foundation (ODF), telephone number 0800226611, or after hours emergency number 0847419484. The ODF website can also be visited at www.odf.org.za. We should all try to encourage an increase in living kidney donation despite cultural misconceptions. This will entail educating and increasing awareness of organ donation in our communities. Potential living donors can contact the National Kidney Foundation of SA (NKFSA) for more information by sending an e-mail to nkfsa@mweb.co.za.

SA has led the world in transplanting deceased HIV-positive donors to HIV-positive recipients, with good preliminary results. This programme has already transplanted a growing number of patients under the leadership of Dr Elmi Muller at Groote Schuur Hospital, Cape Town, SA.

Living-related allograft recipients enjoy a significant graft survival advantage over those who receive deceased donor graft. Living donor transplant has the advantage of being performed with minimum delay. Pre-emptive transplant (transplantation prior to dialysis) is possible.

Evaluation of a living donor would entail:

- compatible donor and recipient blood group

- cross-match between the individuals

- human leukocyte antigen

- typing.

If compatible, the donor is to be scheduled for further evaluation. Contraindications to donation:

- proteinuria/haematuria

- moderately abnormal urological anatomy

- active infection

- chronic active viral infection (hepatitis B or C and/or HIV)

- active malignancy

- history of malignancy - lung, breast, renal, gastrointestinal, haematological, melanoma

- chronic illness, or cardiac, pulmonary, liver, autoimmune, neurological ilnesses

- family history of renal cell cancer

- diabetes mellitus

- significant hypertension

- nephrocalcinosis and bilateral or recurrent kidney stones

- poorly controlled psychosis

- substance abuse

- pregnancy. 


\section{Donor nephrectomy}

Although $50 \%$ of functioning renal mass is removed, post-nephrectomycompensating hypertrophy in the remaining normal kidney returns the GFR to approximately $70 \%$ of baseline at $10-14$ days.

Long-term follow-up studies have not shown progressive loss of GFR over time.

Donor survival was similar to that of the general population matched for age, sex and race. The rate of ESKD in the donor group was lower than that among the general population.

Laparoscopic donor nephrectomy is performed in some transplant centres in SA, which may shorten hospital stay for the donor.

\section{Female donor}

A female donor of child-bearing age should have completed any plans for a family prior to considering kidney donation. There is an increased risk for fetal loss, gestational diabetes, gestational hypertension and pre-eclampsia during pregnancy after kidney donation than in pregnancy in non-donors.

N.B.: Organ trading and organ donation involving any financial transaction between donor and recipient are illegal in SA.

\section{Evaluation of potential renal transplant recipient}

Kidney transplantation is the treatment of choice for ESKD, although there is growing evidence that the dialysis survival rate in SA is higher than in most other countries. Successful kidney transplantation improves the quality of life of most patients compared with dialysis.

Contraindications:

- untreated infection

- malignancy with short life expectancy

- chronic illness with life expectancy $<1$ year

- poorly controlled psychosis

- active substance abuse.

All other patients should be assessed and evaluated by the transplant team to ascertain age suitability for transplantation. Age is no longer considered a contraindication to transplantation. Many patients $>60$ years of age have been transplanted safely. Assessment will focus on fitness of the patient to undergo surgery.

\section{Renal diagnosis}

The type of original kidney disease is not a contraindication to transplantation. Although many diseases recur in the allograft and occasionally lead to graft failure, e.g. focal segmental hyalinosis and IgA nephropathy, no disease recurrence is so common as to preclude a patient from transplant.

\section{Renal function}

There are recommendations for adult candidates for placement on the transplant list when the GFR is $<18 \mathrm{~mL} / \mathrm{min}$ with progressive renal disease.

\section{Nephrectomy}

Pre-transplant nephrectomy of a potential recipient is not recommended. The only exception is autosomal polycystic kidney disease, which may require nephrectomy owing to recurrent symptomatic cyst-related complications or a very large size cystic kidney that makes transplant surgery difficult.

\section{Coronary artery disease (CAD)}

All patients with a high risk for CAD (especially diabetics) usually have a full cardiac assessment by a cardiologist prior to consideration for listing. Cardiovascular disease is the leading cause of death after kidney transplantation.

\section{Cerebrovascular disease}

Older patients and patients with risk factors will be evaluated with carotid Doppler to exclude carotid stenosis. All patients who have had a transient ischaemic attack need to be evaluated fully by a neurologist.

\section{Obesity}

Extremely obese patients (BMI $\left.>35-40 \mathrm{~kg} / \mathrm{m}^{2}\right)$ are excluded from transplantation, unless significant weight loss is achieved.

\section{References}

1. James PA, Oparil S, Carter BL et al. 2014 evidence-based guideline for the management of high blood pressure in adults: Report from the panel members appointed to the Eighth Joint National Committee (JNC 8). JAMA 2014;311(5):507-520. [http://dx.doi.org/10.1001/jama.2013.284427]

2. The ONTARGET Investigators. Telmisartan, ramipril, or both in patients at high risk for vascular events. N Engl J Med 2008;358:1547-1559. [http://dx.doi.org/10.1056/NEJMoa0801317] 